\title{
Comparison of Spin Dynamics in the Cylindrical and Frenet-Serret Coordinate Systems
}

\author{
Alexander J. Silenkd* \\ Bogoliubov Laboratory of Theoretical Physics, \\ Joint Institute for Nuclear Research, Dubna 141980, Russia, \\ Research Institute for Nuclear Problems, \\ Belarusian State University, Minsk 220030, Belarus
}

\begin{abstract}
A comparative analysis of a description of spin dynamics in the cylindrical and Frenet-Serret coordinate systems is carried out. An equivalence of these two systems is shown. A possibility of efficient use of the cylindrical coordinate system for a calculation of spin evolution of particles and nuclei in accelerators and storage rings is caused by an immobility of its coordinate axes relative to stationary detectors.
\end{abstract}

PACS numbers: 29.20.D-, 29.27.Hj

*Electronic address: alsilenko@mail.ru 


\section{INTRODUCTION}

This paper is devoted to a comparative analysis of describing spin dynamics in the cylindrical and Frenet-Serret coordinate systems. The latter is necessary because of the importance of precisely measuring the evolution of particle and nuclei polarizations in accelerators and storage rings. The basic equation for spin motion in electromagnetic fields is a Thomas-Bargmann-Michel-Telegdi (T-BMT) one [1], given in Cartesian coordinates. It is very important to search for electric dipole moments (EDMs) of particles and nuclei in the

experiments prepared for running at storage rings [2]. To calculate the spin dynamics in such experiments, it is necessary to use a generalization of the T-BMT equation, which takes into account the presence of EDM (see Ref. [3] and references therein).

The equations mentioned above determine spin motion in Cartesian coordinates. However, polarized beams of particles and nuclei in accelerators and storage rings move along practically closed trajectories. In accelerator theory, a standard choice for the coordinate system is a Frenet-Serret one (FS), whose axis orientation is fixed by the particle motion. In this case, the equation of spin motion describes the motion of a spin (pseudo)vector with respect to the momentum vector, i.e., the change in the relative orientation of these vectors. This fact complicates a description of the spin dynamics. In the real world, one measures spin orientation relative to the detectors, whose position is fixed in the cartesian coordinates rather than with respect to the momentum vector, whose projections onto all three axes of a given reference frame change. In Ref. [4], an alternative description and exact equations describing the motion of a particle and its spin in the cylindrical coordinate system were found. However, a detailed analysis of differences in the descriptions of spin dynamics in both coordinate systems was not carried out in Ref. [4]. Because the problem of particles and nuclei spin dynamics in accelerators and storage rings is vitally important, such an analysis should be done anyway.

We use the system of units $c=1$. 


\section{COMPARISON OF CYLINDRICAL AND FRENET-SERRET REFERENCE FRAMES}

In a cylindrical coordinate system, the azimuthal angle $\phi$ is given by the particle position. Upon changing a particle azimuth by the angle $d \phi$ the horizontal axes of cylindrical and cartesian coordinate systems turn with respect to each other by the same angle; i.e., the cylindrical coordinate system rotates with respect to the Cartesian one around the $z$ axis with an instantaneous angular velocity $d \phi / d t$ (see Ref. [4]).

The axes of the FS coordinate system are set by the particle trajectory. The orts of this reference frame are directed along the tangent to the trajectory (parallel to the velocity and momentum), in the trajectory plane along the normal to it (parallel to an acceleration vector), and along the binormal perpendicular to these two orts. Relative to the Cartesian coordinate system, an FS one rotates about all three axes, not only around $z$ axis as a cylindrical reference frame. The motion of particles and nuclei in the vertical direction is oscillatory in accelerators and storage rings.

The peculiar features of the cylindrical and FS coordinate systems can be quantitatively described by setting an evolution of a unit vector along the vectors of particle velocity and momentum, $\boldsymbol{N}=\boldsymbol{v} / v=\boldsymbol{p} / p$. From the Lorentz equation

$$
\frac{d \boldsymbol{p}}{d t}=e(\boldsymbol{E}+\boldsymbol{\beta} \times \boldsymbol{B}), \quad \boldsymbol{\beta}=\frac{\boldsymbol{v}}{c}=\frac{\boldsymbol{p}}{\gamma m},
$$

it follows that this vector rotates with an instantaneous angular velocity [4]

$$
\boldsymbol{\omega}=-\frac{e}{\gamma m}\left(\boldsymbol{B}-\frac{\boldsymbol{N} \times \boldsymbol{E}}{\beta}\right) .
$$

The additional (with regard to the rotation of a cylindrical coordinate system) rotation of the FS reference frame relative to the Cartesian one occurs in the presence of nonzero horizontal components of magnetic and/or quasimagnetic fields $\boldsymbol{B}$ and $\boldsymbol{N} \times \boldsymbol{E}$. The relationship between an angular velocity of $\boldsymbol{N}$ vector rotation and relative motion of cylindrical and Cartesian coordinate systems found in Ref. [4] is not trivial. Let us denote the projection of any vector onto a horizontal plane by the symbol $\|$. Since the vector $\boldsymbol{N}$ is directed along the tangent to the trajectory, the change in the particle azimuth in this plane equals an angle between two horizontal projections of this vector, which can be denoted as $\boldsymbol{N}_{\|}$and $\boldsymbol{N}_{\|}^{\prime}$. An infinitesimal angle $d \phi$ characterizing the change in the particle azimuth is given by 
the following expression:

$$
d \phi=\frac{\left(\boldsymbol{N}_{\|} \times \boldsymbol{N}_{\|}^{\prime}\right) \cdot \boldsymbol{e}_{z}}{\left|\boldsymbol{N}_{\|}\right| \cdot\left|\boldsymbol{N}_{\|}^{\prime}\right|}=\frac{\left(\boldsymbol{N}_{\|} \times d \boldsymbol{N}_{\|}\right) \cdot \boldsymbol{e}_{z}}{\left|\boldsymbol{N}_{\|}\right|^{2}},
$$

where $d \boldsymbol{N}_{\|}=\boldsymbol{N}_{\|}^{\prime}-\boldsymbol{N}_{\|}$is an infinitesimal vector. The instantaneous rate of the azimuth change is found to be [4]

$$
\dot{\phi} \equiv \frac{d \phi}{d t}=\frac{\left(\boldsymbol{N}_{\|} \times \dot{\boldsymbol{N}}_{\|}\right) \cdot \boldsymbol{e}_{z}}{\left|\boldsymbol{N}_{\|}\right|^{2}}=\omega_{z}-o
$$

where

$$
o=\frac{\left(\omega_{x} N_{x}+\omega_{y} N_{y}\right) N_{z}}{1-N_{z}^{2}}=\frac{\left(\omega_{\rho} N_{\rho}+\omega_{\phi} N_{\phi}\right) N_{z}}{1-N_{z}^{2}} .
$$

The components of the $\boldsymbol{\omega}$ vector are fixed by Eq. (2).

Equations (3) and (44) are exact. This is shown in [4] by considering an example of a particle running the loop whose normal is deviated from the $z$ axis by some angle. Taking into account a correction due to quantity $o$ allows an exact description of the particle motion

projected onto a horizontal plane. However, the quantity $o$ is usually rather small. If the horizontal plane coincides with the plane of unperturbed particle motion, this quantity can usually be neglected (see analysis in [4]).

\section{EQUATION OF SPIN MOTION}

In all reference frames considered so far, the spin motion is precessional. The effects due to a spin-tensor interaction (see [7-10] and references therein) are considered in the present paper. Let the particle with electric and magnetic dipole moments moves in the electromagnetic field. The equation describing the motion of its spin in the Cartesian coordinate system looks as follows [3]]:

$$
\begin{aligned}
\frac{d \boldsymbol{s}}{d t}= & \boldsymbol{\Omega} \times \boldsymbol{s}, \quad \boldsymbol{\Omega}=\boldsymbol{\Omega}_{M D M}+\boldsymbol{\Omega}_{E D M}, \\
\boldsymbol{\Omega}_{T-B M T}= & -\frac{e}{m}\left[\left(a+\frac{1}{\gamma}\right) \boldsymbol{B}-\frac{a \gamma}{\gamma+1} \boldsymbol{\beta}(\boldsymbol{\beta} \cdot \boldsymbol{B})\right. \\
& \left.-\left(a+\frac{1}{\gamma+1}\right)(\boldsymbol{\beta} \times \boldsymbol{E})\right], \\
\boldsymbol{\Omega}_{E D M}= & -\frac{e \eta}{2 m}\left(\boldsymbol{E}-\frac{\gamma}{\gamma+1} \boldsymbol{\beta}(\boldsymbol{\beta} \cdot \boldsymbol{E})+\boldsymbol{\beta} \times \boldsymbol{B}\right),
\end{aligned}
$$

where the quantities $\Omega_{M D M}$ and $\boldsymbol{\Omega}_{E D M}$ make contributions of electric and magnetic dipole moments, respectively. The same look is acquired by the quantum mechanical equations of spin motion for particles with spins 1/2 [5] and 1 [6] after transition to the classical limit. 
An angular velocity of spin rotation in the cylindrical coordinate system is obtained by subtracting the quantity $\Omega$ from $\dot{\phi} \boldsymbol{e}_{z}$. Upon neglecting the correction $o$, it is given by the expression

$$
\begin{array}{r}
\boldsymbol{\Omega}^{(c y l)}=-\frac{e}{m}\left\{a \boldsymbol{B}-\frac{a \gamma}{\gamma+1} \boldsymbol{\beta}(\boldsymbol{\beta} \cdot \boldsymbol{B})\right. \\
+\left(\frac{1}{\gamma^{2}-1}-a\right)(\boldsymbol{\beta} \times \boldsymbol{E})+\frac{1}{\gamma}\left[\boldsymbol{B}_{\|}-\frac{1}{\beta^{2}}(\boldsymbol{\beta} \times \boldsymbol{E})_{\|}\right] \\
\left.+\frac{\eta}{2}\left(\boldsymbol{E}-\frac{\gamma}{\gamma+1} \boldsymbol{\beta}(\boldsymbol{\beta} \cdot \boldsymbol{E})+\boldsymbol{\beta} \times \boldsymbol{B}\right)\right\},
\end{array}
$$

where $a=(g-2) / 2$. A comparison of Eqs. (5) and (6) shows that the horizontal projections of vectors $\boldsymbol{\Omega}$ and $\boldsymbol{\Omega}^{(c y l)}$ coincide.

To find the angular velocity of spin motion in the FS coordinate system, it is necessary to subtract an angular velocity of rotation of the $\boldsymbol{N}$ vector from $\boldsymbol{\Omega}$. This way, in this reference frame, an angular velocity of spin rotation is found to be [3]:

$$
\begin{array}{r}
\boldsymbol{\Omega}^{(F S)}=-\frac{e}{m}\left[a \boldsymbol{B}-\frac{a \gamma}{\gamma+1} \boldsymbol{\beta}(\boldsymbol{\beta} \cdot \boldsymbol{B})+\left(\frac{1}{\gamma^{2}-1}-a\right)(\boldsymbol{\beta} \times \boldsymbol{E})\right. \\
\left.+\frac{\eta}{2}\left(\boldsymbol{E}-\frac{\gamma}{\gamma+1} \boldsymbol{\beta}(\boldsymbol{\beta} \cdot \boldsymbol{E})+\boldsymbol{\beta} \times \boldsymbol{B}\right)\right] .
\end{array}
$$

Equation (7) is commonly used in the literature to describe a spin motion in accelerators and storage rings.

Of course, Eq. (77) is more compact. However, this compactness is achieved owing to the fact that the deviation angles from the vertical line for the FS coordinate system axes change with time, while the radial and azimuthal axes of the cylindrical coordinate system always belong to a horizontal plane. Therefore, Eq. (7) can in particular create the illusion that, under regular conditions $(\boldsymbol{\beta} \cdot \boldsymbol{B}=0)$, the efficiency of impact of vertical and radial fields on a spin is identical. However, when $\boldsymbol{E}=0$ and the EDM is neglected, the ratios $\Omega_{\rho}^{(c y l)} / B_{\rho}$ and $\Omega_{z}^{(c y l)} / B_{z}$ differ in fact by $(a \gamma+1) /(a \gamma)$ times. For leptons (an electron and a muon) this ratio can be very large. The reason is that, to determine an observable effect, the motion of tangential and normal axes of the FS coordinate system should be added to the spin motion in this coordinate system. Once this factor is taken into account, both coordinate systems give an equivalent description of the spin motion. As an example demonstrating the necessity of correct taking into account a spin rotation around the horizontal coordinate axes, let us mention the experiment that measured the anomalous magnetic moment of a muon [11]. In 
this experiment, the muon momenta satisfied the condition $1 /\left(\gamma^{2}-1\right)=a$. The presence of a weak radial magnetic field and vertical electric one, which compensates for the impact of the former on the particle motion, leads to a certain increase in the angular velocity

of spin rotation: $\sqrt{\Omega_{z}^{2}+\Omega_{\rho}^{2}}$ instead of $\Omega_{z}$. A naive usage of Eq. (77) for calculating $\Omega_{z}$ without taking into account the motion of tangential and normal axes of the FS coordinate system leads to an expression that substantially differs from the correct result given by Eq. (6). Note that a thorough elimination of magnetic field inhomogeneities in the experiment [11] allowed a significant reduction in the radial magnetic field; its contribution to the final angular velocity of spin rotation in this latter experiment was negligible.

\section{CONCLUSIONS}

The comparative analysis of a description of spin dynamics in the cylindrical and FrenetSerret coordinate systems carried out in this paper has demonstrated that it is possible to use both reference frames. The advantage of the FS coordinate system is a mathematical apparatus thoroughly developed on its basis for describing spin evolution of particles and nuclei in accelerators and storage rings. However, the cylindrical coordinate system may as well be efficiently used for that purpose. Its advantage is the absence of motion of the coordinate axes relative to the plane of unperturbed motion of particles/nuclei and, consequently, with respect to the stationary detectors.

This work was supported by the Belarusian Republican Foundation for Fundamental Research (Grant No. Ф14D-007).

[1] L. H. Thomas, "The Motion of the Spinning Electron," Nature (London) 117 (2945), 514 (1926); L. H. Thomas, "The Kinematics of an Electron with an Axis," Philos. Mag. 3 (13), 1 (1927); V. Bargmann, L. Michel and V. L. Telegdi, "Precession of the Polarization of Particles Moving in a Homogeneous Electromagnetic Field," Phys. Rev. Lett. 2 (10), 435 (1959).

[2] T. Fukuyama, "Searching for new physics beyond the Standard model in electric dipole moment," Int. J. Mod. Phys. A 27 (16), 1230015 (2012).

[3] T. Fukuyama and A. J. Silenko, "Derivation of generalized Thomas-Bargmann-Michel-Telegdi 
equation for a particle with electric dipole moment," Int. J. Mod. Phys. A 28 (29), 1350147 (2013).

[4] A. J. Silenko, "Equation of spin motion in storage rings in the cylindrical coordinate system," Phys. Rev. ST Accel. Beams. 9 (3), 034003 (2006).

[5] A. J. Silenko, "Quantum-mechanical description of the electromagnetic interaction of relativistic particles with electric and magnetic dipole moments," 48 (8), 9, (2005).

[6] A. J. Silenko, "Quantum-mechanical description of spin-1 particles with electric dipole moments," Phys. Rev. D 87 (7), 073015 (2013).

[7] A. J. Silenko, "Tensor electric polarizability of the deuteron in storage-ring experiments," Phys. Rev. C 75 (1), 014003 (2007).

[8] A. J. Silenko, "Potential for measurement of the tensor polarizabilities of nuclei in storage rings by the frozen spin method," Phys. Rev. C 80 (4), 044315 (2009).

[9] V. G. Baryshevsky and A. J. Silenko, "Potential for the measurement of the tensor electric and magnetic polarizabilities of the deuteron in storage-ring experiments with polarized beams," J. Phys. Conf. Ser. 295 (1), 012034 (2011).

[10] A. J. Silenko, "High precision description and new properties of a spin-1 particle in a magnetic field," Phys. Rev. D 89 (12), 121701(R) (2014).

[11] G. W. Bennett et al (Muon (g-2) Collaboration), "Final report of the E821 muon anomalous magnetic moment measurement at BNL," Phys. Rev. D 73 (7), 072003 (2006). 